\title{
Production of necropsy reports by medical staff using a computer system
}

\author{
AG BROWN, CL BERRY \\ Department of Morbid Anatomy, The London Hospital, London
}

SUMMARY A computer system for reporting necropsies by medical staff is described, which includes diagrams and allows typing to be kept to minimum owing to the format and the development of a lexicon. The system has been in use for a year, during which time it has been modified.

The studies of Cameron et al emphasised the importance and value of necropsies in modern medicine. ${ }^{1}$ Cameron also outlined the value of the necropsy in medical audit ${ }^{23}$ at a time when inaccurate death certification data may be used to determine, in part, the distribution of health resources. The fall in necropsies, documented by Cameron but evident to most pathologists, has been caused by many factors including misplaced clinical optimism about the accuracy of newer diagnostic techniques. The increase in the number of coroners' necropsies, which, according to the report by the working party on medical aspects of death certification convened by the Royal Colleges of Physicians and Pathologists contribute little to audit, has further decreased the amount of accurate data.

The pathologist has a clear responsibility to clarify exactly what necropsies have to offer clinical practice. Poor communications seem to be a central part of this problem ${ }^{4}$ and delays in reporting are a considerable irritation. Not unreasonably in view of the priorities in a busy office, necropsy reports are often placed at the end of the line after surgical biopsies, cytology reports, correspondence, and papers. A "late" report may not influence the notes, and where a clear discrepancy between clinical diagnosis and necrospy findings exists the clinical view may prevail in the final summary.

To minimise delay in getting gross necropsy reports to the relevant clinician we devised a system whereby medical staff could produce a report directly, avoiding the use of a secretary and thus another stage in the process. These reports were usually completed on the day of examination; subsequent histology and SNOP coding was done using the systems described previously, ${ }^{5}$ with subsequent modifications. ${ }^{6}$

Accepted for publication 13 August 1985

\section{Material and methods}

We used an Apple II + computer and Applesoft Basic language in the initial work but decided that the optional use of diagrams should be built into the system to reduce the amount of description required to complete a report. Pictures generated by computer occupy large amounts of storage space and operating memory, and the program was therefore moved to an Apple III with a five megabyte "PROFILE" hard disk. This allowed all of the programs for text input, the picture library, print program, data storage, ando support routines to be stored on one disk, with rou-? tines being moved into and out of memory as required in automatic predefined sequence. More storage space was available and access time greatly reduced.

Although the Apple III has an adequate memory for quite a large program, we decided that as the reporting of necropsies was subdivided anatomically the program would be written as a series of small linked units reflecting this division. With this technique only the unit in use needs to be resident in the memory and on completion is replaced by the next unit; the user does not need to control the running of the program as the answers to certain questions can help decide which units to use. For example, if the subject of the report is a man the unit will bypass those units associated with women. This approach also means that individual units can be modified without introducing major changes into the overall format of the system, and that rewriting in a different language for a machine with a smaller memory is possible.

As one of the main considerations for the program was minimal training of staff the method of text entry and manipulation was constructed for simplicity. The input of text was limited to 60 characters per line. On reaching the end of a line further input is halted until the "return" key is pressed, up to which point it is 
possible to edit the line being entered. Previously entered lines can be modified by using the edit routines provided at the end of each unit.

The amount of description required for a particular feature clearly varies, and we therefore decided that no limit should be put on the amount of space allowed. The screen scrolls to allow further text entry where necessary. The final format displayed to the user is the same as that seen on the printed report. After the editing sequence the data are saved and the next program unit is called into the memory.

The program starts by requesting general information: necropsy examination number; surname; christian names; age; sex; race; hospital record number; date of death; date of necropsy; hospital consultant; prosecutor; and previous surgical numbers. Necropsy number, surname, and sex must be entered before the program will proceed. At two points in the list the user is asked to confirm that the details are correct and is given the opportunity to make amendments. When these details are completed space is provided to enter the clinical history. The program now takes the user through the reporting procedure system by system.

For each system a standard format is used, which begins by asking for the weights of the various organs associated with it. The program automatically provides units of grams, centimetres, and millilitres to ensure that a uniform system is used for all reports. An opportunity is then offered to bypass the entire system if there are no features requiring comment. If this option is selected only the weights and a standard phrase showing that the system contained no important abnormality is printed in the report. If the system is to be commented on each structure for comment is displayed in turn. The screen displays the name of the structure and the two vertical lines delineating 60 characters per line. At the bottom of the screen a list of numbered phrases applicable to the structure is shown, and any number of these phrases may be used within the description by selecting a number from the keyboard. All phrases can be updated and the lexicon enlarged with little amendment to the program. When all of the systems have been run space is made available for a summary of the necropsy findings.

A menu of available pictures is then displayed. When a picture is selected it is loaded from the library on disk and displayed on the screen. At the bottom of the screen three numbered squares and three circles of increasing dimensions are displayed, and by means of a point cursor, which is moved using the "arrow" keys and selection of the required number, these shapes may be used to delineate areas for comment. To try a shape for size on the picture a second selection of the same number is required to fix it in posi- tion; any other key will automatically erase it. The arrow keys may also be used to draw any other required shape. Text can be added to the picture by typing in the normal manner, and an erase mode is also available, again using the arrow keys to move the cursor. On completion of a picture the amended version is saved on the disk and the menu redisplayed.

Three copies of the report are printed automatically, and we have ensured that it is impossible to enter another report in our system until this is completed. Reports are printed in a 60 column format with a maximum of 60 lines to a page; each page has the necropsy examination number at the top. The first page shows all the general details listed above, and on this page only the headings are in normal density print. The information is in enhanced print, enabling easier retrieval of the information required for identifying cases. These details are succeeded by the clinical summary and necropsy summary. The various systems are then printed, each one beginning with the weights and followed by the separate structures under their appropriate headings. If a feature was not commented on the heading for that structure is omitted from the printout. The program looks ahead to calculate the space available on the current page while printing, and if there is not enough room for a complete description of a structure to fit in it moves on to a new page to avoid splitting the text. On completion of the text three copies of any pictures used are printed.

No permanent disk storage is used as the entry of a subsequent report reuses the data storage files after erasing the information they contain. This is not a fixed feature of the program and could easily be modified to allow long term storage of the reports on disk.

\section{Discussion}

The system has been operating for just over a year, and several modifications have been suggested by users, including an ability to vary the system order in the report, so that major findings in terms of clinical relevance can be presented first. The ten junior staff who used the system varied in their reaction to keyboard use, although all agreed that it was likely to prove a skill that all laboratory workers should acquire. The diagram facility is underused, no doubt because it is clumsy: if a "mouse" or "light pen" could be added to the system the facility would probably be used more.

We decided that it was probably desirable to store the data until the histology could be added as modification of the report may be desirable on some occasions. Changes already made include the provision at the start of each system of a "general fea- 
tures" category that can be used to enter relevant information that does not easily fall into any of the structures provided by the program. At regular intervals a review is carried out of the reports generated so far, and from these the most commonly used descriptive phrases are extracted and used to increase the content of the lexicon.

It is the interested clinical staff who undertake high number of necropsies and who attend necropsies on their patients in any case who have commented favourably on the scheme. Even if we are preaching to the converted then we can still be satisfied with prompter reporting, an increased awareness of the familiarity with computer use among junior staff, and less pressure on the office staff while surgical and cytological workloads increase. These may be sufficient rewards in themselves, but it is also true that the quality of reports has probably increased, although this is difficult to measure objectively.

Previously developed computer aided necropsy reporting systems have focused mainly on the storage and retrieval of necropsy diagnoses. ${ }^{7-10}$ This has been achieved using a keyword approach to code the data and access to a large computer with its associated storage space. In our system we concentrated on achieving a standard yet flexible necropsy report on a micro computer and used a previously developed system $^{5}$ for the storage of coded diagnoses.
References

${ }^{1}$ Cameron HM, McGoogan E. A prospective study of 1152 hospital autopsies. 1. Inaccuracies in death certification. $J$ Pathol 1981(a);133:273-84.

${ }^{2}$ Cameron HM. The autopsy past and present. J R Coll Physicians Lond 1984;18:236-9.

${ }^{3}$ Cameron HM. The autopsy- illusion and reality. In: Cameron HM, ed. Pathology annual, Vol 18. Connecticut: AppletonCentury-Crofts, 1983:333-45.

${ }^{4}$ Cameron HM, McGoogan E. A prospective study of 1152 hospital autopsies. 2 Analysis of inaccuracies in clinical diagnosis and their significance. Hum Pathol 1981(b);133:285-300.

${ }^{5}$ Swettenham KV, Nickols CD, Berry CL. Computer programs in histopathology and record keeping. J Clin Pathol 1982;35:40-4.

${ }^{6}$ Swettenham KV, Nickols CD, Berry CL. Computer programs in cytology reporting and record keeping. J Clin Pathol 1983;33:856-9.

${ }^{7}$ Paplanus SH, Shepard RH, Zvargulis JE. A computer-based system for autopsy diagnosis storage and retrieval without numerical coding. Lab Invest 1969;20:139-46.

${ }^{8}$ Sutinen S, Koskinen P, Vastamaki R. A method for automatic storage and retrieval of alphabetic autopsy diagnoses coded by computer. Lab Invest 1974;30:762-6.

${ }^{9}$ Brust A, Doerre W, Hopker WW, Kayser K. A standardized autopsy-protocol-problems and solutions. Virchows Arch (Pathol Anat) 1974;364:1-14.

${ }^{10}$ Takahashi M, Masuda M, Isiglo K, Matsumoto T. A pathology data management system (PADAMS 7810) for the interactive storage and retrieval of autopsy records. Methods In Med 1979;18:151-7.

Requests for reprints to: Professor CL Berry, Department of Morbid Anatomy, The London Hospital, London E1 1BB, England 